\title{
Digital Image Correlation for Cement-based Materials and Structural Concrete Testing
}

\author{
Tambusay, A. ${ }^{1,2}$, Suryanto, B..$^{*}$, and Suprobo, . $^{2}$
}

\begin{abstract}
This paper presents the application of a low-cost digital image correlation (DIC) system for automated crack mapping. The system employed makes use of a conventional digital camera, a remote image recording controller, and an open-source digital image correlation MATLAB software Ncorr. To showcase the potential of the proposed system, two case studies involving general structural and material testing were undertaken. In the first series, the DIC system was used to study the fracture processes in a reinforced concrete beam and to investigate the influence of key input parameters on the spatial quality of strain maps obtained from the DIC analysis. In the second series, the application of the DIC method was expanded to assist in the study of complex multiple micro-crack formations in ductile cement composite testing. It is shown that the strain maps obtained from the DIC technique have a close resemblance to the actual crack patterns.
\end{abstract}

Keywords: Crack mapping; DIC; low-cost; Ncorr; strain field.

\section{Introduction}

Digital image correlation (DIC) is a non-contact measurement technique that can be used to track the full-field strain and displacement of a deforming object [1]. With this technique, strain and displacement can be measured across the entire surface of a large object without a prior knowledge of key locations of interest, as normally required in traditional measurements such as using strain gauges or transducers. The risk of missing critical areas of importance can, therefore, be minimised, making it particularly useful in a situation where the regions of importance could not be easily identified.

Since its first introduction in the 1980s [2, 3], DIC has undergone considerable advancement and progressed to the point where it is finding use in a broad range of applications across multiple disciplines, including civil engineering. Current use of DIC in this field is, however, still limited to niche applications and its popularity has been partly hampered by the high initial cost of the system, typically in the range US\$20k-70k [4]. Accordingly, the use of DIC has been largely confined to specialist research laboratories and test institutes.

\footnotetext{
${ }^{1}$ Institute for Infrastructure and Environment, Heriot-Watt University, Edinburgh, UNITED KINGDOM.

${ }^{2}$ Department of Civil Engineering, Sepuluh Nopember Institute of Technology, Surabaya, INDONESIA.

* Corresponding author; email: b.suryanto@hw.ac.uk
}

Note: Discussion is expected before July, $1^{\text {st }} 2020$, and will be published in the "Civil Engineering Dimension", volume 22, number 2, September 2020.

Received 29 January 2020; revised 17 February 2020; accepted 04 March 2020.
McCormick and Lord at the National Physical Laboratory in the UK [5], for example, demonstrated the potential of DIC to capture the full extent of concrete cracking which is barely visible to the naked eye. Gencturk and co-workers [6] used DIC in their full-scale testing of prestressed concrete beams and noted that the strains measured from the DIC were comparable to conventional gauges, although the strain profiles reported in their study appear to fall short of sufficient detail and visual description. Reddy and Subramaniam [7] used the commercial VIC-2D software to study the complex fracture behaviour around a notch in fibre reinforced concrete specimens. The importance of careful consideration of accuracy and resolution was emphasised.

In this paper, a relatively inexpensive system is devised and optimised, with the aim to promote a more widespread use of DIC within the civil and structural engineering field. The main intention was to develop a system that exhibits the characteristics of robustness, ease of use, adoption for general material and structural testing, and low-cost. Bearing these aspects in mind, it was decided to use a conventional digital camera as the main recording instrument and an open-source image correlation software known as Ncorr [8-11] which runs on a widely used programming platform MATLAB $B$.

This paper will provide a discussion of the capabilities of the proposed system in two case studies: a reinforced concrete beam under four-point bending and a dog-bone shaped, ductile cement composite known as the engineered cementitious composite (ECC) [12-14]. In the first case study, parametric analysis was undertaken to study the influence of key input parameters on the spatial quality and resolution of the strain maps produced by the DIC. The 
second case study was chosen to test the robustness of the proposed system in capturing successive formation and widening of multiple micro-cracks generated during testing.

\section{Digital Image Correlation}

Digital Image Correlation (DIC) is a non-contact technique that can be used to track the full-field displacement and strain on the surface of a specimen. In essence, this technique works by comparing two images, with a reference image captured before the deformation and a second image captured after the deformation. It uses an image registration technique for mapping the local and global displacements, from which strains can be derived.

To obtain the full deformation field, the image of an object needs to be divided into small subsets (see, for example, Figure 1). Each subset must have a unique pattern, so the algorithm can distinguish subset similarity, effectively matching a target subset block with a reference subset block.

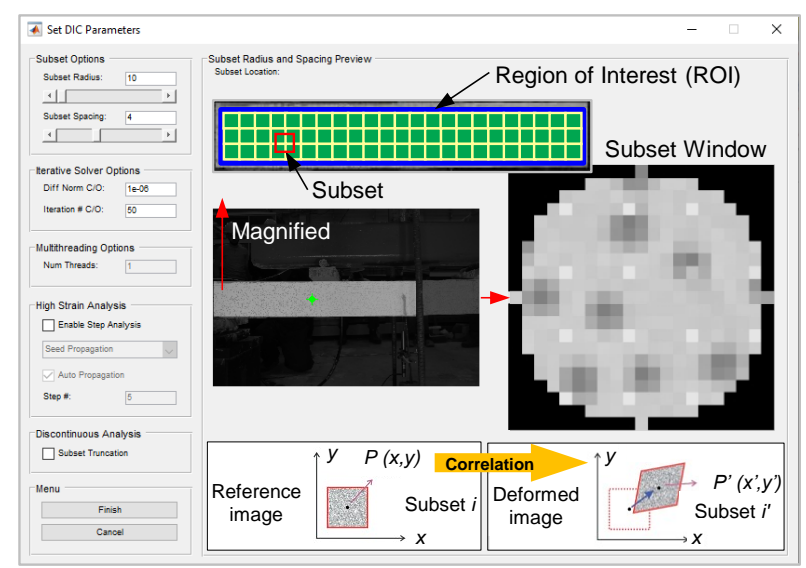

Figure 1. Reference Image Divided into Image Subsets.

Based on the location of the centre-point of the subset in both the reference and target images, the local displacement vector is produced and the deformation within each subset is computed. This process is repeated for all subset blocks over the entire surface of the object to obtain the full displacement map, from which strains can be derived [15].

\section{Ncorr}

Ncorr is an open-source 2D digital correlation MATLAB ${ }^{\circ}$ program [16]. In Ncorr, digital images are represented by an assemblage of small subsets which are controlled by two parameters: subset radius, r, and subset spacing, s; when $r$ is equal to $0.5 \mathrm{~s}$, subsets will be in the form of contiguous circles with a radius of r. In Ncorr, the deformation inside each subset is assumed to be homogeneous. A linear first-order coordinate transformation is used [8]. The displacement field of a subset is computed using a cost function (normalized cross-correlation), which is then further refined using the Inverse Compositional GaussNewton method [17]. Ncorr then uses the Reliability Guided method to obtain the displacement values for all subsets, which are then converted to strain fields by employing the Green-Lagrangian strain formulation [8]. Given that the calculation of the strain field involves differentiation, it is sensitive to noise. To rectify this problem, Ncorr implements the leastsquares plane fit method [8].
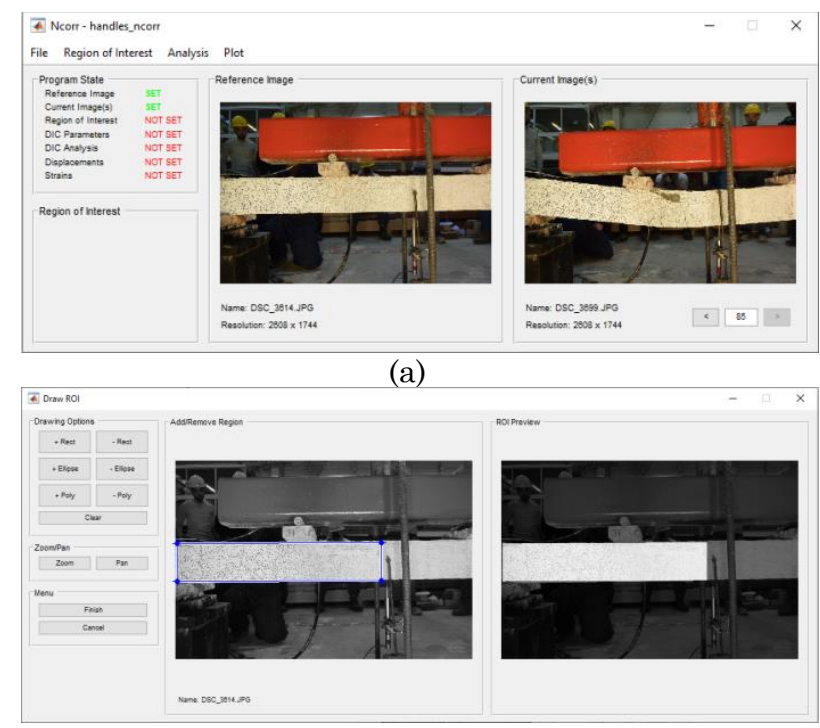

(b)

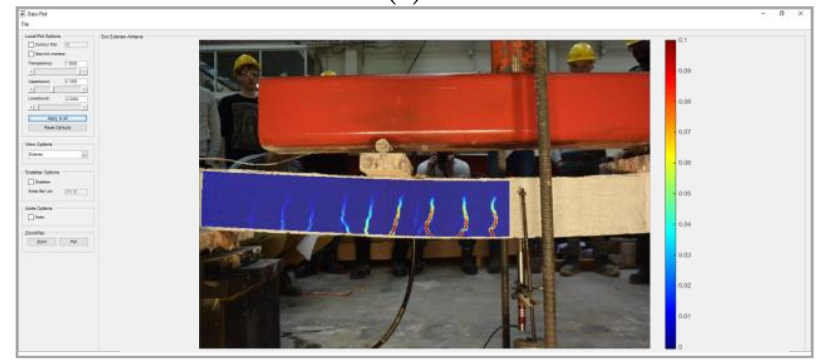

(c)

Figure 2. (a) Ncorr main terminal. (b) Region of Interest (ROI). (c) Computed strain profile.

Apart from these features, Ncorr has the capabilities to handle multi-thread computation, large deformation, rigid-body deformation and strong discontinuity. For more detailed information on the algorithms used in the software, the reader is referred to $[8,17]$.

Figure 2(a) displays the screenshot of the main user interface terminal of Ncorr. To perform a DIC analysis, a reference image of an object needs to be first uploaded and this will be then displayed in the left-hand side window of the main terminal. Then, a series of images taken during testing needs to be uploaded and these individual images can then be checked from the right-hand side window. Following this, the Region of Interest (ROI) needs to be defined, as highlighted in the right-hand side window in Figure 2(b), in order to mark the area where the DIC analysis is needed. Figure 2(c) shows an example of the strain maps obtained from a DIC analysis. 
In the following section, two series of DIC analysis are presented to verify the robustness of the proposed system in constructing the full-field strain and faithful crack maps.

\section{Case Study 1: Concrete Beam}

A reinforced concrete beam with overall dimensions of $100 \times 150 \times 2000 \mathrm{~mm}^{3}$ was tested under four-point bending. The schematic of the beam geometry and steel reinforcement layout are presented in Figure 3. The top and bottom longitudinal reinforcements were each provided by two $8 \mathrm{~mm}$ bars. Transverse reinforcement was provided in the form of rectangular closed $8 \mathrm{~mm}$ links at a spacing of $75 \mathrm{~mm}$. The concrete at the time of testing had a strength of 38.4 MPa.

The beam was simply supported over a span of $1.7 \mathrm{~m}$. Two-point loads were applied on the top of the beam at $0.7 \mathrm{~m}$ centres via a spreader beam, with the load applied at the centre of this spreader beam using a hand-operated hydraulic jack. The beam deflection was monitored at midspan using a displacement transducer placed under the beam. Prior to testing, random dots were manually drawn using a black marker to give a random pattern with a sharp contrast for the DIC analysis.

The loading process was stopped at $5 \mathrm{kN}$ intervals for crack observations. In addition to this manual process, strain/crack mapping was done using the DIC. During testing, images were taken every $0.5 \mathrm{kN}$ using an 18.4MP Nikon 1 J4 mirrorless digital camera. The camera was placed onto a tripod and positioned at a distance of $600 \mathrm{~mm}$ from the front face of the beam. Communication with the camera was established using a smartphone via the Nikon's wireless mobility utility application.

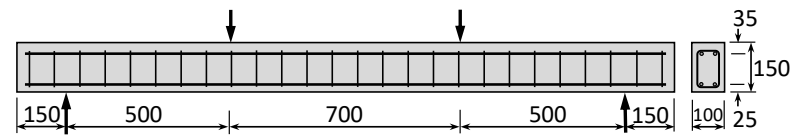

Figure 3. Beam Geometry and Reinforcement Layout. Dimension is in $\mathrm{mm}$.

Figure 4 presents the longitudinal strain plots over the half left of the beam at different stages of loading. For comparative purposes, the load-deflection response of the beam and the corresponding crack pattern over the right half are presented in Figures 5 and 6, respectively. It is evident from Figure 4 that the first stage of loading resulted in a uniform strain contour due to the low strain values experienced at this stage of loading. Thereafter, a development of parallel strips of high strain can be found at the bottom of the beam over the centre span, which is in general agreement with the flexural cracks shown in Figure 6. The extent and prominence of the strips become more evident with increasing load implying that they are of indicative of the actual crack opening and geometry. It is noteworthy that the compressive strain at the top of the beam is shown in dark (black) colour due to its relatively small value and the wide range of strain values employed (i.e. from a small negative value to $10 \%$ ).

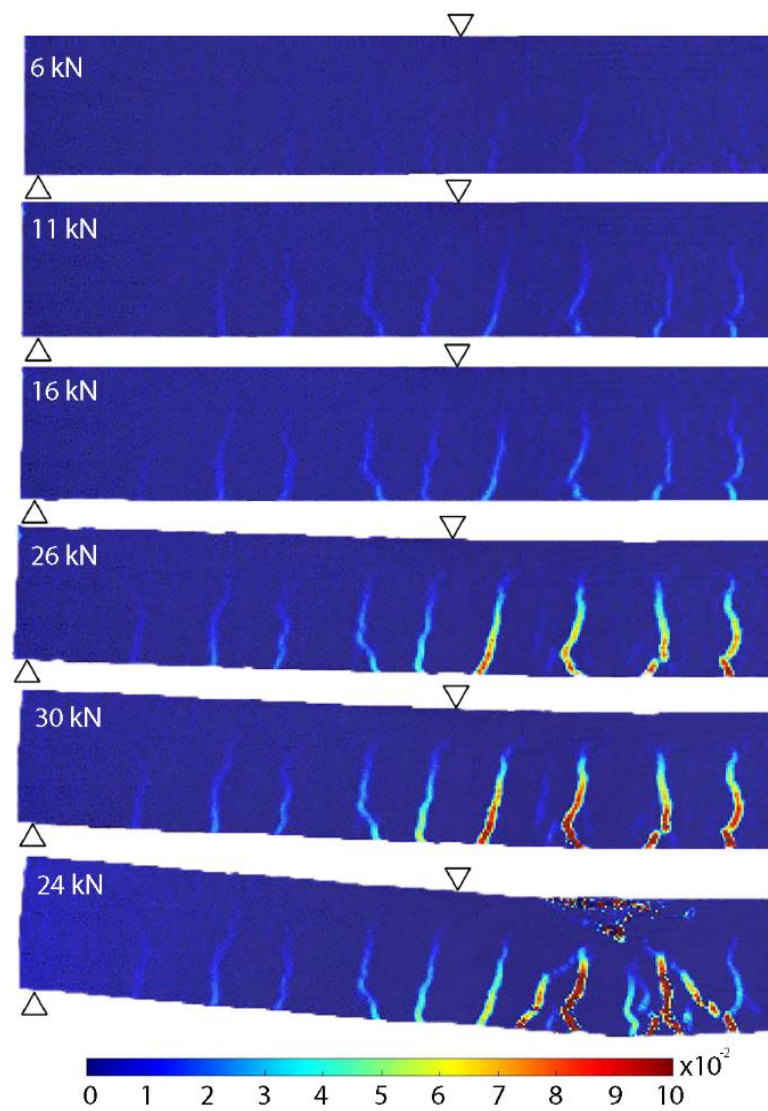

Figure 4. Longitudinal Strain Plots Obtained from the DIC Analysis. The subset radius $r=10$, subset spacing $s=4$ and strain radius $=1$.

At load in excess the yield capacity (i.e. $26 \mathrm{kN}$ ), the strain strips over the centre span display an increase in value, indicating crack widening resulting from yielding of the tension bars at crack locations. These strain values at each strip also visibly vary and increase toward the bottom of the beam, which is indicative of changing in crack width.

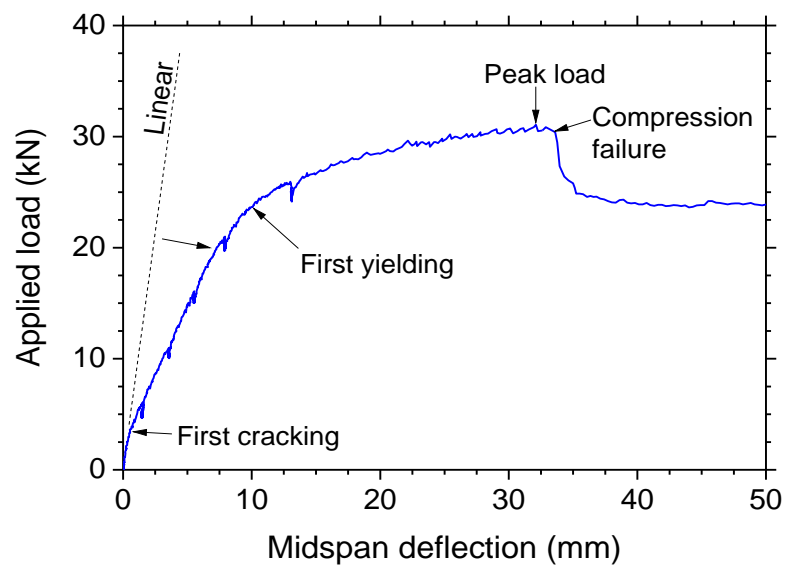

Figure 5. Load-deflection Response 


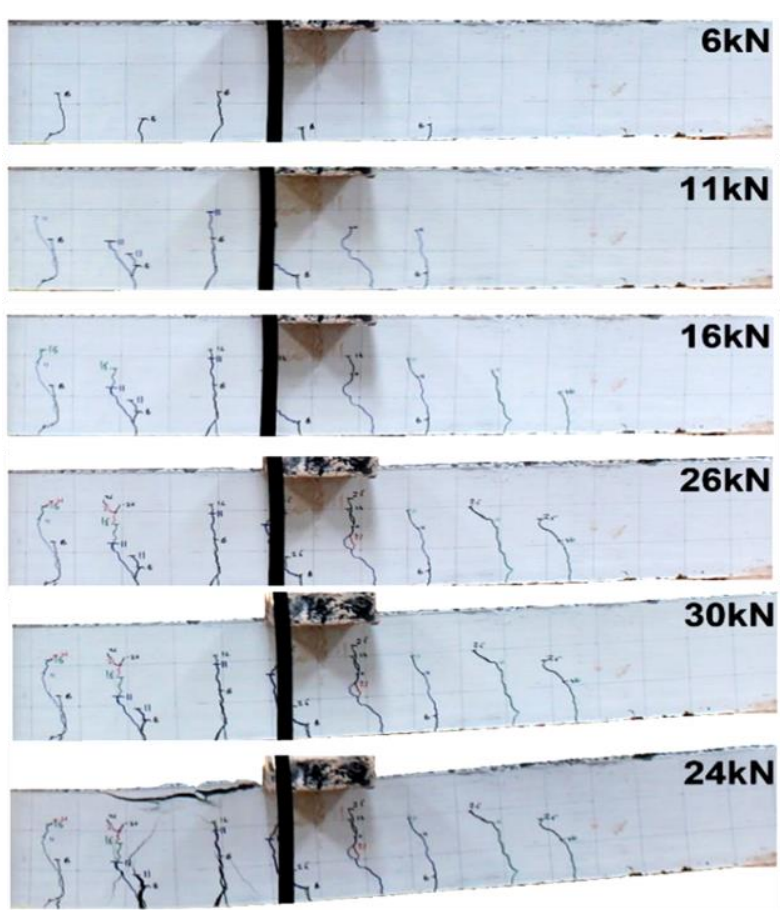

Figure 6. Observed Crack Patterns under Different Stages of Loading. Failure was due to Crushing of the Concrete on Top.

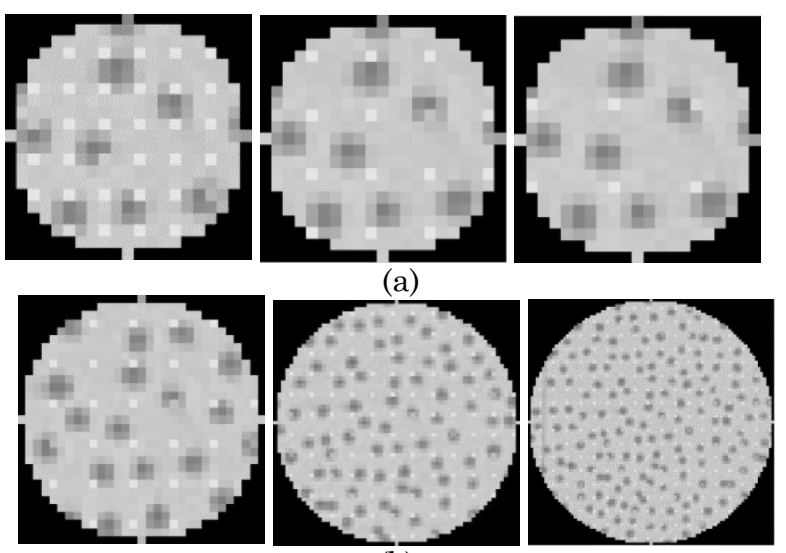

(b)

Figure 7. Subset Window with Different Input Parameters: (a) $r=10$ and $s=2,4,6$; (b) $s=4$ and $r=15,30$, and 45. The white dots indicate the centre of each subset, whereas the grey dot represent the hand-drawn dotted pattern.

To investigate the influence of two main parameters in the DIC analysis (subset radius, $r$, and subset spacing, s) on spatial quality and resolution of the strain field, a series of sensitivity analyses were performed. Figures 8 and 9 present the influence of subset spacing, s, on computation time and spatial quality; the subset radius, $r$, was taken as 10 for illustrative purposes. It is evident from Figures 8, 9(a) and (b) that as $s$ is increased from 2 to 6 pixels, it results in a large reduction in computation time with no visible degradation in image quality, highlighting the beneficial effect of increasing subset spacing on computational time cost. Nevertheless, increasing $\mathrm{s}$ further results in a reduction in image resolution and in an undesirable smoothing effect, particularly when $s$ is larger than $r$ (see, for example, Figures 9(c) and (d)). Based on this, a subset spacing between $20 \%$ and $60 \%$ of the subset radius is recommended.

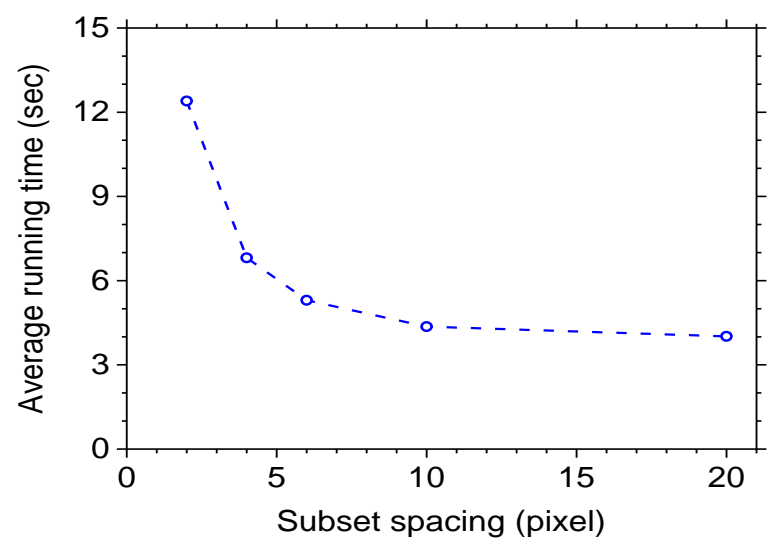

Figure 8. Relationship between Running Time per Image and Subset Spacing. Analysis Performed on a Workstation with 12 Intel Xeon E5-1650 3.2GHz processors, 40GBDDR3 (1600MHz) RAM and a 7200rpm hard drive.

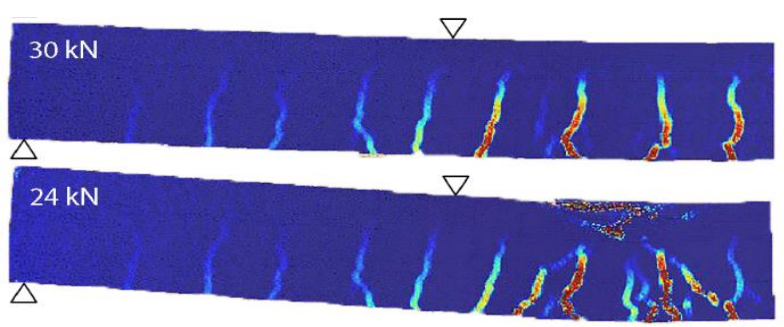

(a)
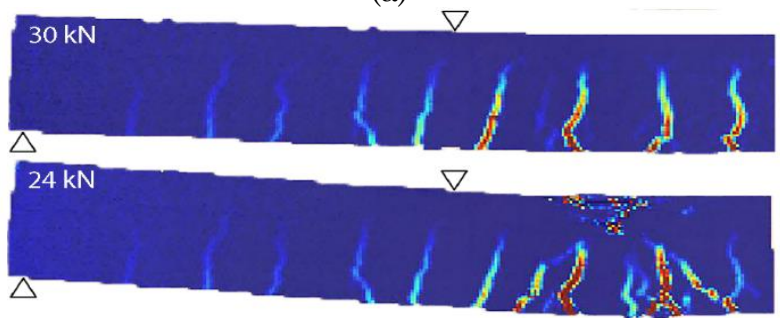

(b)

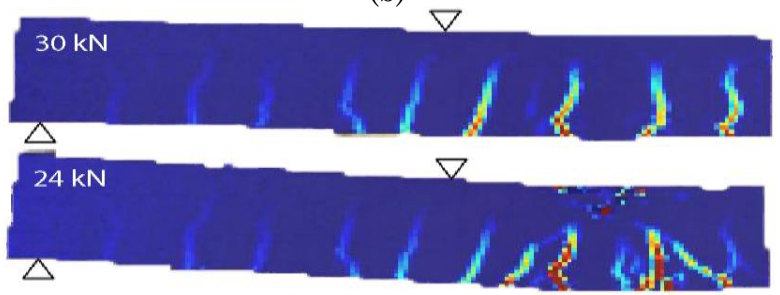

(c)

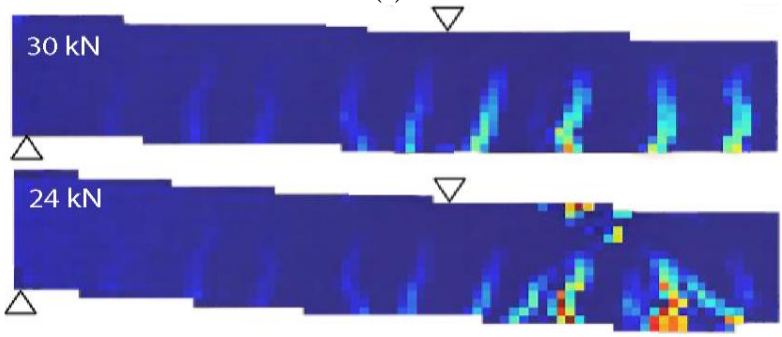

(d)

Figure 9. Influence of Subset Spacing on Image Quality: (a) $s=2$; (b) $s=6$; (c) $s=10$; and (d) $s=20$. The subset radius $r=$ 10 (constant). 
The influence of subset radius, $r$, on the quality of the DIC strain images is presented in Figures 10(a)-(d). The subset spacing, s, was fixed at 4 pixels and a range of values was chosen for $\mathrm{r}$, including 10, 15, 30 and 45 pixels. The minimum value of $r$ was determined visually from the average size and average spacing of the dotted pattern (see Figures 7(a) and (b)); at $\mathrm{r}=10$, it was judged from the subset window that the subset still retains an adequate level of contrast and detail.

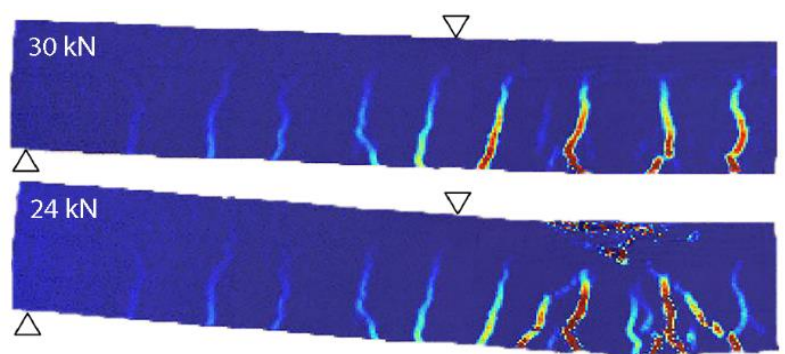

(a)
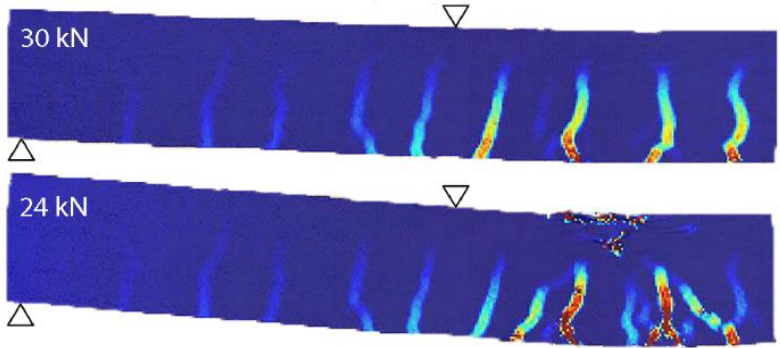

(b)

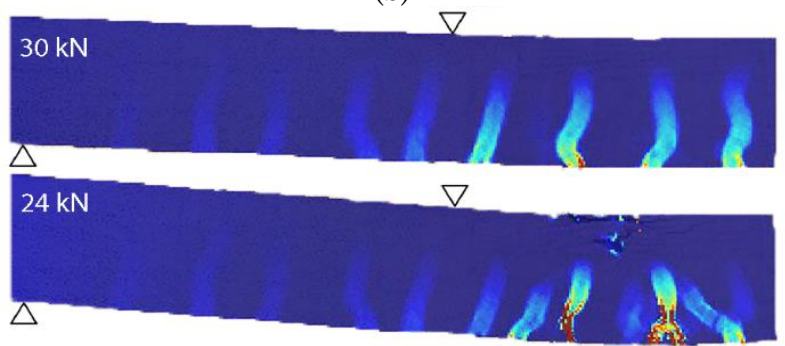

(c)
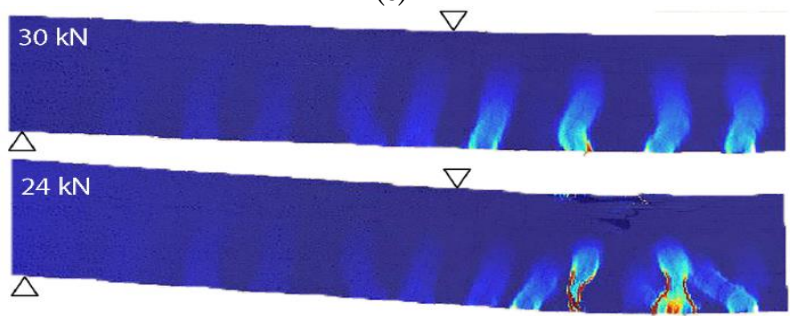

(d)

Figure 10. Influence of Subset Radius on Image Quality: (a) $r=10$; (b) $r=15$; (c) $r=30$; and (d) $r=45$. The subset spacing $\mathrm{s}=4$ (constant).

Concerning the results presented in Figure 10, it is apparent that, as with the influence of $s$, increasing $r$ has the effect of altering the image quality and introducing a smoothing effect which is seen to worsen the definition of the flexural cracks forming along the length of the beam. This is due to the assumption made in the DIC algorithm that the deformation inside each subset is assumed to be homogeneous. In this particular example, the best quality of the reconstructed image was accomplished with $r=10$. No detectable noise was apparent.

\section{Case Study 2: ECC Coupon}

To demonstrate the potential of the system for material testing, the result of a dog-bone shaped ECC coupon specimen, with overall dimensions displayed in Figure 11, is presented. This was the first ECC produced in Indonesia using locally available materials. The ECC mix was optimised, so as it exhibits strain-hardening response under tension.

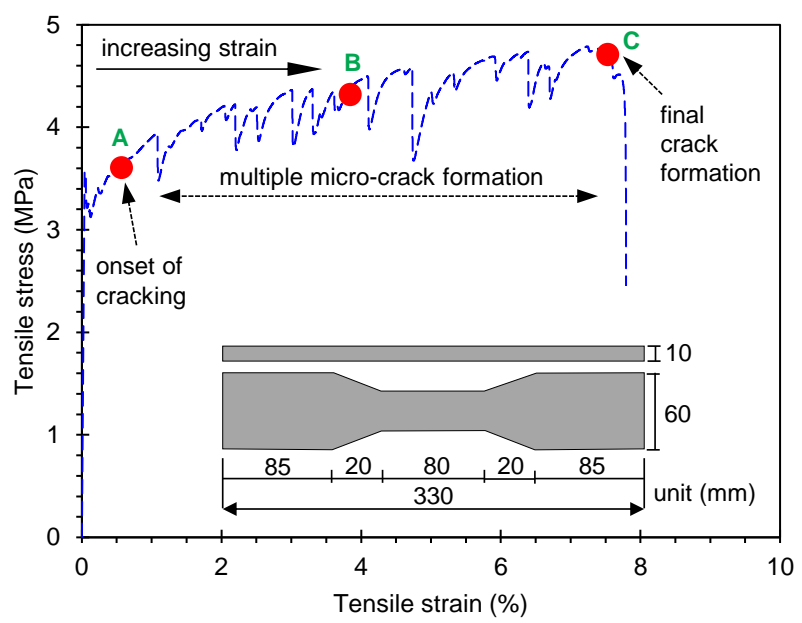

Figure 11. Tensile Stress-strain Response and Specimen Geometry.

Tensile testing was performed using a universal testing machine under a displacement rate of 0.5 $\mathrm{mm} / \mathrm{min}$. Each sample was held at each end using a pair of grips slotted at each side of the sample. The mean tensile strain on the central part of the sample was measured using a displacement transducer mounted via a pair of plastic holders placed at the opposing ends of the central neck of the sample (see Figure 12).

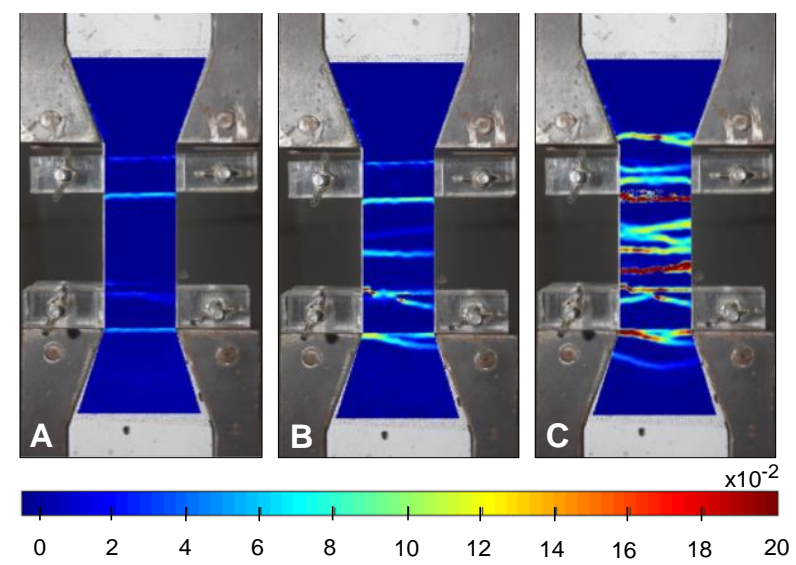

Figure 12. DIC Strain Maps at Stages Labelled as A, B and $\mathrm{C}$ in Figure 11. The subset radius $r=30$, subset spacing $\mathrm{s}=$ 6 and strain radius $=3$. The plastic holders at the opposing ends of the central neck of the dog-bone specimen are used to mount a LVDT at the back surface. 
Figure 11 presents the tensile stress-strain response of the coupon. Tensile stresses were determined by dividing load cell readings from the test machine by the cross-sectional area of the narrower (central) section (30×10 (thick) $\mathrm{mm} 2$ ), whereas the tensile strains within this region were determined from the displacement transducer readings. It is evident that first cracking occurred at a stress of approximately 3.5 $\mathrm{MPa}$ and this was then followed by notable fluctuations in tensile stress with increasing tensile strain, clearly indicating a strain hardening response resulting from successive formation of multiple cracking. This continues until the average strain attained a value of $\sim 8 \%$.

To perform automated crack mapping, the front surface of the dog-bone was sprayed white and a random, closely spaced dotted pattern was drawn using a marker. The DIC analysis was then performed following that used in the first case study. In this case, however, a higher image resolution (6000×4000 pixels) was used to ensure distinctive pixel intensity and to overcome the difficulty when dealing with very small crack widths typical of an ECC. The subset radius was taken as 30 pixels, while the subset spacing as 6 pixels following the recommended range in the first case study.

With reference to Figure 12, it is evident that the initiation of subtle crack development in excess of the tensile cracking of the ECC matrix is reasonably captured, with each high-strain strip showing a relatively uniform strain value indicative of microcrack of comparable width. As the ECC coupon is further loaded, a more discernible strain profile is apparent, with new near-parallel strip formation evident due to optimised fibre bridging. This indicates successful reproduction of successive formation of micro-cracking in the ECC. This cracking process continues until a crack localisation is eventually formed, which leads to a sudden drop in tensile stress and hence the failure of the ECC coupon.

\section{Concluding Remarks}

1. The DIC system presented in this study is shown to provide good capabilities for performing automated crack mapping in general structural and material testing. The system is shown to produce crack maps which are in good agreement with traditional methods.

2. Appropriate subset inputs are important to avoid the risk of decreasing spatial resolution. It is shown that while increasing subset spacing has the beneficial effect of reducing computational cost, it may lead to a reduction in image resolution, particularly when the subset spacing is larger than the subset radius. A value between $20 \%$ and $60 \%$ of the subset radius is recommended.
3. Increasing the subset radius has the effect of introducing a blurring effect and hence reducing the image quality.

4. The challenge of constructing reasonable strain maps in specimen attributed with very small crack widths can be tackled with proper image resolution and speckle size.

Overall the results have supported the conviction that the DIC system presented in this paper is straightforward to perform in terms of analysis process and instrumentation required. It presents itself as an attractive tool for general material and structural testing.

\section{Acknowledgements}

The authors wish to acknowledge the financial support from the Ministry of Research, Technology and Higher Education of the Republic of Indonesia (Grant No. 5/EI/KP.PTNBH/2019). The authors also wish to thank Mr. J.L. Fox and S. Cassar for their assistance in part of the work.

\section{References}

1. Schreier, H., Orteu, J.J., and Sutton, M.A., Image Correlation for Shape, Motion and Deformation Measurements, s.l.: Springer, United States, 2009.

2. Peters, W. and Ranson, W.F., Digital Imaging Techniques in Experimental Stress Analysis, Optical Engineering, 21(3), 1982, pp. 213427213427.

3. Sutton, M.A., Wolters, W.J., Peters, W.H., Ranson, W.F., and McNeil, S.R., Determination of Displacements using an Improved Digital Correlation Method, Image and Vision Computing, 3(1), 1983, pp. 133-139.

4. Mesocos s.r.o., DIC Measurements in Engineering Applications, Prague, 2015.

5. McCormick, N. and Lord, J., Digital Image Correlation for Structural Measurements, ICE Publishing, London, United Kingdom, 2012, pp. 185-190.

6. Gencturk, B., Hossain, K., Kapadia, A., Labib, E., and Mo, Y-L., Use of Digital Image Correlation Technique in Full-scale Testing of Prestressed Concrete Structures, Measurement, 47, 2014, pp. 505-515.

7. Reddy, K.C. and Subramaniam, K.V.L., Experimental Investigation of Crack Propagation and Post-cracking Behaviour in Macrosynthetic Fibre Reinforced Concrete, Magazine of Concrete Research, 69(9), 2017, pp. 467-478.

8. Blaber, J., Adair, B., and Antoniou, A., Ncorr: Open-source 2D Digital Image Correlation Matlab Software, Experimental Mechanics, 55(6), 2015, pp. 1105-1122. 
9. Suryanto, B., Tambusay, A., and Suprobo, P., Crack Mapping on Shear-critical Reinforced Concrete Beams using an Open-Source Digital Image Correlation Software, Civil Engineering Dimension, 19(2), 2017, pp. 93-98.

10. Tambusay, A., Suryanto, B., and Suprobo, P., Visualization of Shear Cracks in a Reinforced Concrete Beam using the Digital Image Correlation, International Journal on Advanced Science, Engineering and Information Technology, 8(2), 2018, pp. 573-578.

11. Suryanto, B. and Staniforth, G., Monitoring the Shear Fatigue Response of Reinforced Concrete Beams Subjected to Moving Loads using Digital Image Correlation, Civil Engineering Dimension, 21(1), 2019, pp. 6-12.

12. Li, V.C., Engineered Cementitious Composites (ECC), Springer, Germany, https://link.springer. com/book/10.1007/978-3-662-58438-5.
13. Kanda, T., Tomoe, S., Nagai, S., Maruta, M., Kanakubo, T., and Shimizu, K., Full-scale Processing Investigation for ECC Precast Structural Element, Journal of Asian Architecture and Building Engineering, 5(2), 2006, pp. 333-340.

14. Suryanto, B., Reynaud, R., and Cockburn, B., Sectional Analysis of Engineered Cementitious Composite Beams, Magazine of Concrete Research, 70(22), 2018, pp. 1135-1148.

15. McCormick, N. and Lord, J., Digital Image Correlation, materialstoday, 13(2), 2010, pp.52-54.

16. Ncorr version 1.2. Available at http://www.ncorr. com/download/ncorr_v1_2_1.zip

17. Harilal, R. and Ramji, M., Adaptation of Opensource 2D DIC Software Ncorr for Solid Mechanics Applications, $9^{\text {th }}$ International Symposium on Advanced Science and Technology in Experimental Mechanics, New Delhi, India, November 1-6, 2014. 